PROKLA-Redaktion

\section{Editorial: Politik mit der Inneren (Un-)Sicherheit}

Mit dem globalen „Krieg gegen den Terrorismus" hat sich nicht nur die geopolitische Weltkarte, sondern auch das innenpolitische Terrain der westlichen Demokratien erheblich verändert. Nach den Anschlägen vom 11. September 2001 haben staatliche Überwachungsmaßnahmen und Repressionspolitiken in Europa und Nordamerika erheblich zugenommen. Geheimdienstliche und „sicherheits"politische Maßnahmen sollen vermutete oder echte Bedrohungen antizipieren, aufspüren und verhüten, bevor sie als tatsächliche Gefahr überhaupt eintreten: Nicht eine manifeste Bedrohung, sondern schon das plausibel angenommene Risiko einer solchen hält die Sicherheitsmaschinerie in Gang. Hand in Hand damit geht der Abbau bürgerlicher Schutzrechte. Angesichts der mittlerweile alltäglich gewordenen öffentlichen Debatten um präventive Spitzelmaßnahmen à la Bundestrojaner, Gefangenenfolter und den Abschuss ziviler Flugzeuge stellt sich die dringliche Frage nach den Grenzen des Rechtsstaates, sowie nach den sozialen Widerständen gegen eine zunehmend autoritäre und präventionsstaatliche Sicherheitspolitik.
Staatliche Repressions- und Überwachungspolitiken hatten freilich auch schon lange vor den Anschlägen auf das World Trade Center Konjunktur - und obwohl sie meist im Gewand des Anti-Terrorismus-Diskurses daherkamen, stellten sie stets auf oppositionelle Bewegungen, AktivistInnen und Intellektuelle aller Art ab. Bereits in den 1960er und 1970er Jahren rüsteten sich die demokratischen Rechtsstaaten der westlichen Welt mit neuen Gesetzen, Technologien und (Bürger-)kriegsstrategien gegen die Studenten- und Arbeiterbewegungen und gegen militante Befreiungsbewegungen. Konservative und sozialdemokratische Politiker zogen dabei häufig am gleichen Strang.

In Westeuropa gaben die sozialen Proteste, die in der Revolte von 1968 gipfelten, den Anlass für autoritäre Schübe. Das politische System Frankreichs reagierte schon in den 1960ern mit einem präsidialen Ruck - hier wirkte sicherlich auch noch die Kriegsführung in Algerien zurück ins kolonialistische "Mutterland“. In Italien wurden Ende der 1970er in wenigen Monaten mehr als 10.000 basisgewerkschaftlich-linksradikale Aktivisten (Stichwort: Autonomia) für
Jahre weggesperrt. Hunderte gingen ins Exil. Das post-francistische Spanien behielt Teile des unter Franco eingerichteten Repressions- und Folterapparats bei und setzt diese bis zum heutigen Tage gegen die Autonomie-Bestrebungen im Baskenland ein. In den USA, der ältesten bürgerlichen Demokratie der Welt, reagierte die Regierung Johnson in den 1960er Jahren auf die erstarkenden Proteste gegen den Vietnamkrieg mit der illegalen Überwachung von FriedensaktivistInnen. Nur kurze Zeit später begann die paramilitärische Zerschlagung der Black Panther Bewegung durch COINTELPRO, das staatliche "Counterintelligence Program against Black-Hate Groups". Die Liste ließe sich beliebig verlängern.

In Deutschland beschloss nach einer zehnjährigen Diskussion und umfangreichen sozialen Protesten eine Große Koalition aus CDU und SPD im Jahr 1968 die sogenannten Notstandsgesetze, die es ermöglichten zahlreiche Grundrechte einzuschränken, darunter etwa das Briefgeheimnis und das Post- und Fernmeldegeheimnis. Nur wenige Jahre darauf verabschiedete die von Bundeskanzler Willy Brandt geführte Regierungskoalition von SPD und FDP den sogenannten Radikalenerlass, der die ideologische und rechtliche Grundlage für die jahrzehntelang währende Praxis des Berufsverbots bildete. Auf das Jahr 1976 und die immer noch sozial-liberale Koalition geht der „Terrorismusparagraph" 129 a zurück, der bis heute der Einschüchterung und Ausforschung politischer Akti-
vistInnen dient, derzeit etwa im Umfeld der globalisierungskritischen Bewegung. Unter dem Vorwand der Terrorismusverfolgung, den die Aktionen der RAF lieferten, gab es seit den frühen 1970er Jahren immer wieder Versuche, linke Gruppierungen und Protestbewegungen aus dem „Verfassungsbogen“ auszuschließen und zu kriminalisieren.

Polizeiliche Abwehrreaktionen und Prävention sowie geheimdienstliche Kontrollversuche im Moment „sozialer Unordnung ${ }^{\prime 6}$ stellen also alles andere als ein Novum oder eine Ausnahmeerscheinung dar. Vielmehr finden sich lange Kontinuitäten: Einmal geschaffene Gesetze und polizeiliche Einrichtungen bleiben über Jahrzehnte bestehen und bilden einen konstitutiven Bestandteil staatlicher Herrschaftssicherung - auch unter demokratisch-rechtsstaatlichen Bedingungen. Sie werden ergänzt durch neue polizeiliche Behörden, Mechanismen der Zusammenarbeit und Methoden der Überwachung. Das Projekt der Herrschaftssicherung nimmt dabei stets historisch-spezifische Formen an, die vor dem Hintergrund der jeweiligen gesellschaftlichen Kräfteverhältnisse und sozio-ökonomischen Konstellationen zu sehen sind. Eine Analyse der jüngeren sicherheitspolitischen Entwicklungen muss daher zum einen historisch informiert sein und zum anderen die Besonderheiten des gegenwärtigen Kapitalismus und dessen politische Formationen berücksichtigen.

Ebenso gilt es zu bedenken, dass es in zahlreichen gesellschaftlichen Be- 
reichen auch zu einer Ausweitung von Rechten und zur gesetzlichen Anerkennung chemals kriminalisierter Gruppen gekommen ist: So hat sich zum Beispiel die Rechtslage von Schwulen und Lesben erheblich verbessert, Diskriminierungen aufgrund von Geschlecht, Alter oder Rassismus wurden verboten, neue Gesetze ermöglichen Individuen, ihre entsprechenden Rechte gegen staatliche Behörden und Arbeitgeber besser zu schützen. Auf der institutionellen Ebene haben sich das Verbandsklagerecht und der Konsumentenschutz entwickelt; Bürger und Bürgerinnen können sich - zumindest auf kommunaler Ebene - zunehmend in politische Entscheidungsverfahren einbringen. Nicht zuletzt gab es in den vergangenen Jahren starke soziale Proteste gegen die neoliberale Politik und es formieren sich Ansätze einer sozialistischen Linken, die den Anspruch erhebt, zur Entwicklung einer nach-kapitalistischen Gesellschaft beizutragen. Die aktuelle Neuformierung der Herrschaftssicherung vollzieht sich also nicht als Entwicklung hin zu einem autoritären Staat nach klassischem Muster, in dem staatlich autoritäre Repression mit herrschaftlich instrumentalisierten Sekundärtugenden und konformistischer Unterwerfungsbereitschaft auf Subjektebene zusammenläuft.

Vor diesem Hintergrund stellt sich die Frage, wie die Gesetzesverschärfungen und die Ausweitung exekutiver Praxen und Befugnisse nach dem sicherheitspolitischen Dammbruch vom 11. September 2001 einzuord- nen sind: Handelt es sich um eine politische Konjunktur, die sich mit einer Änderung der politischen Kräfteverhältnisse auch schnell wieder ändern kann? Erleben wir „nur" eine weitere Welle von Sicherheitsgesetzen und repressiven Maßnahmen, die sich nicht substanziell von ihren Vorgängern der 1960er, 1970er und $1980 \mathrm{er}$ unterscheidet? Oder kommt den Überwachungstechniken und Unterdrückungsstrategien der vergangenen Jahre eine neue Qualität $z u$ ? Und wenn ja, worin besteht diese und wie wird sich dies in Zukunft auswirken?

Um Antworten auf diese Fragen zu finden, lassen Alexander Klose und Hubert Rottleuthner in ihrem Beitrag die bundesdeutsche Geschichte der Gesetzesverschärfungen Revue passieren und ordnen sie in ihre jeweiligen gesellschaftspolitischen Kontexte ein. Andreas Fisahn überprüft drei theoretische Deutungsversuche auf ihre empirische Geltung: die Theorien des Ausnahmezustands, des Überwachungsstaates und des autoritären Etatismus. Er entscheidet sich tendenziell für den autoritären Etatismus als Dispositiv, das es den verschiedenen Fraktionen der Herrschenden ermöglicht, ihre teilweise auch konfligierenden Interessen pluralistisch durchzusetzen, sich den demokratischen Ansprüchen der Restgesellschaft aber ,repressiv tolerant" zu entziehen.

Eine neue Dimension staatlicher Überwachung scheint sich dabei vor allem durch die Nutzung never Informationstechnologien zu eröffnen.
Zwar versuchte auch schon dic Volkszählung von 1987 das Ideal des „gläsernen Bürgers" in die Realität zu übersetzen - und wurde dabei durch eine breite soziale Bewegung und Boykotthaltung sabotiert -, doch die massenhafte Verbreitung und Nutzung des Personal Computers und die Popularisierung des Internet seit Mitte/Ende der 1990er Jahre, z.B. be der Buchung von Reisen, beim Emailen und Online-Banking, auf der eigenen Website oder in Internetforen, macht die massive Erhebung sensibler persönlicher Daten durch staatliche Behörden so einfach und attraktiv wie nie zuvor. Darüber hinaus haben Sicherheitsbehörden nun die technischen Möglichkeiten, Informationen in einem Maße zu speichern, auszuwerten und auszutauschen, von denen sie noch vor wenigen Jahren kaum zu träumen gewagt hätten. Der Widerstand gegen den jüngsten staatlichen Datensammelwahn komm derzeit vor allem von den Gerichten. Andreas Fisahn erinnert daran, dass das Bundesverfassungsgericht sowohl dem Großen Lauschangriff als auch der Online-Durchsuchung von Computern enge Grenzen gesetzt und die verdachtsunabhängige und massenhafte Kennzeichenerfassung und -speicherung gar für verfassungswidrig erklärt hat.

Auch in den USA wird der Konflikt um den Abbau von Grundrechten eher in den Gerichten - und in den Medien - als auf der Straße ausgetragen. So entschied der US-Supreme Court im Juni dieses Jahres, dass der unter George W. Bush verabschiedete
Military Commissions Act 2006 in Teilen verfassungswidrig sei. Die Einrichtung von speziellen Militärtribunalen sei nicht mit der Verfassung vercinbar; auch Guantánamo-Häftlinge hätten das Recht auf eine Anhörung vor einem ordentlichen Gericht. Dic Bürgerproteste gegen die menschenverachtende Haft-, Folter- und Verschleppungspraxis der Regierung Bush bleiben dagegen weiterhin marginal und werden durch rechtliche Taschenspielertricks ins Abseits gedrängt. So zeichnen viele Städte und Universitäten in den USA sogenannte „Free Speech Zones" aus, in denen DemonstrantInnen ihre Meinung kund tun dürfen - das bedeutet allerdings, dass alle anderen Orte „offlimits" sind: eine offensive Containment Policy („Eindämmungspolitik"), die das First Amendment der US-amerikanischen Verfassung faktisch außer Kraft setzt. In der Bundesrepublik ist diese Praxis auf Grund des spezifischen deutschen Versammlungsrechts übrigens schon immer der autoritäre Normalzustand gewesen. Kaum ein anderer Staat verlangt von "seinen Demonstranten“, dass eine öffentliche Kundgebung ordnungsamtlich vorangemeldet und dabei individuelle Personen und Verantwortlichkeiten schriftlich festgelegt werden.

So sehr man sich aus bürgerrechtlicher Perspektive über die (derzeit noch) funktionierende Kontrolle der Exekutive durch die Judikative freuen mag, so dringend ist doch auch die Frage nach der politischen Bewertung dieses Phänomens geboten. 
Zum einen scheint es mehr als bedenklich, wenn bürgerliche Freiheitsrechte ,nur noch" am seidenen $\mathrm{Fa}$ den eines politisch besetzten Obersten Gerichts oder eines parteienproportional besetzten Verfassungsgerichtshofs hängen. Zum anderen gilt es eine vereinfachende Idealisierung der Gerichte als „Hüterinnen der Demokratie $^{6 /}$ zu vermeiden. Ist és doch genau die Aufgabe der Judikative, das Handeln der Exekutive $\mathrm{zu}$ prüfen und mit den vorhandenen Regelungen in Einklang zu bringen. Das schafft einerseits die für ein verrechtlichtes Handeln notwendige Konsistenz und Kohärenz; andererseits wird damit vor allem auch Legitimität für das gesamte politische System produziert. Nicht selten, so stellen Alexander Klose und Hubert Rottleuthner fest, lehnt das Bundesverfassungsgericht die neuen sicherheitspolitischen Vorstöße im ersten Durchgang ab, belehrt den Gesetzgeber aber zugleich, wie das ,gerade noch Mögliche" verfassungskonform umgesetzt werden kann.

In der Bevölkerung scheint die geradezu allgegenwärtige Erfassung persönlicher Daten zumindest ein Unwohlsein auszulösen. Zwar behauptete der „Spiegel“ kürzlich, „die Ängste vor dem totalitären Staat seien, besonders bei den Jüngeren, verblasst. Die Debatten um Online-Durchsuchungen und staatliche Übergriffe lassen sie ziemlich kalt" (Der Spiegel vom 30.7.2007, S. 132). Doch Umfragen des Eurobarometers von 2008 stützen diese Einschätzung nicht. Im Gegenteil: Sie verweisen auf den gleichbleibend hohen Stellenwert, den
Datensicherheit bei der europäischen Bevölkerung genießt. In der Tat lassen sich auch Beispiele dafür finden. So etwa der Fall des Internetforums StudivZ: Als der Betreiber der Website 2007 Nutzerdaten an Werbefirmen weitergeben wollte, protestierten betroffene NutzerInnen vehement und verhinderten das Vorhaben. ${ }^{1}$ Im September 2007 demonstrierten in Berlin mehrere tausend Menschen aus dem gesamten Bundesgebiet unter dem

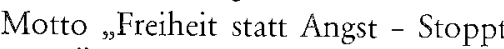
den Überwachungswahn" gegen die ausufernde Überwachung durch Wirtschaft und Staat (vgl. http:// www.vorratsdatenspeicherung.de).

Eine kontinuierlich wahrnehmbare, offensive und politische Massenbewegung gegen die informationstechnologische Überwachungspolitik und das dahinter stehende Staatsverständnis lässt allerdings noch auf sich warten.

In der Tat müsste sich eine solche Basisbewegung nicht nur gegen die Verschärfung von Strafgesetzen, Polizeipraxen und Überwachungsmaßnahmen wenden - auch wenn das sicherlich bereits ein Gewinn wäre -, sondern diese als Bestandteil der neoliberalen Transformation aller Gesellschaftsbereiche kritisieren. Denn erst dann würden auch die Ursachen und Dynamiken auf die politische Agenda gesetzt, die den autoritären Tendenzen der vergangenen Jahre zu Grund liegen. Loïc Wacquant stellt

1 Andreas Busch: Kein Ende der Privatheit: Auch jungen Internetnutzen ist Datenshutz wichtig, WZB Mitteilungen, N wichtig, WZB-Mitteilungen, Nr. 120, Juni
2008 , S. 27-29. in seinem Beitrag den Zusammenhang zwischen der dramatischen $\mathrm{Zu}$ nahme der Gefängnispopulation in den USA und der Reorganisation des Arbeitsmarktes her. Die Logik der Einkerkerungspolitik, so Wacquant, erschließt sich nur, wenn man sie im Kontext der Prekarisierung ganzer Bevölkerungsschichten, der Einführung repressiver Workfare-Maßnahmen und der systematischen Durchsetzung eines Niedriglohnsektors untersucht. Auch John Kannankulam diskutiert die sicherheitspolitischen Veränderungen der letzten Jahre aus einer staatstheoretischen und politökonomischen Perspektive und fragt, inwieweit sich die neuen Kontrollund Überwachungsstrategien als Bestandteil postfordistischer Umwälzungsprozesse interpretieren lassen.

Andreas Fisahn weist in diesem Kontext auf die spezifische Rolle der EU als neoliberaler Schrittmacher hin. Mit ihrer bestenfalls ,staatendemokratischen" Verfassung produziert die EU in allen möglichen Politikberechen - und eben auch sicherheitspolitisch - sogenannte Sachzwänge, die von den noch eher parlamentarischdemokratisch verfassten Mitgliedsstaaten umgesetzt werden müssen. In der BRD werden aktuell postfaschistische, auf die Vorgaben der West-Alliierten zurückgehende Gewaltenteilungsstrukturen geschliffen: die Trennung zwischen Militär nach außen und Polizei nach innen, die zwischen Bundesorganen (Bundespolizei, BKA) und Länderpolizeien und die zwischen Polizei und Geheimdiensten. Dabei wird auch mit EU-
Angleichung bzw. gemeinsamer EUPolitik und den daraus sich ergebenden "Verpflichtungen" argumentiert. Noch unter Schily und Rot-Grün wurde der Bundesgrenzschutz zur Bundespolizei umdeklariert. Nun soll mit dem im Bundeskabinett bereits beschlossenen neuen BKA-Gesetz eine Behörde entstehen, die nicht nur aus der LINKEN als ,geheim ermittelnde Staatspolizei" (Ulla Jelpke) sondern auch von liberaler Seite als "Bundessicherheitshauptamt ${ }^{65}$ (Burkard Hirsch) mit deutlichem historischen Bezug auf die Struktur der entsprechenden Behörde im NSDeutschland scharf kritisiert wird. Was heißt das praktisch für kritische Gesellschaftstheorie und außerparlamentarisches Engagement? Ganz konkret hat der Fall von Andrej $\mathrm{H}$. in Deutschland gezeigt, dass Repression gegen kritische Sozialwissenschaft unter dem Label „Terrorismus" bereits Realität ist (siehe die PROKLA Editorials der Nummern 148 und 149). Tatsächlich hatte $\mathrm{H}$ mit seinem akademischen KnowHow über sozialen Ein- und Ausschluss und Verdrängungsprozesse im Zusammenhang mit Stadtentwicklungspolitiken die ,Systemgrenze zwischen kritischer Wissenschaft und kritischer politischer Praxis überschritten und sich öffentlich innerhalb der globalisierungskritischen Bewegung engagiert. Das muss schon gereicht haben, um bei der Staatssicherheit in die Kategorie des sogenannten Gefährders zu rutschen: Wissenschaft plus Aktivismus ist gleich Terrorismus, so lautete die 
Gleichung der Risikoexperten. Und im Zuge des G-8 Gipfels im Sommer 2007 wurde deutlich, dass die Protes te gegen den Abbau sozialer Rechte, gegen eine menschenverachtende $\mathrm{Mi}$ grationspolitik und gegen weltweite Militärinterventionen mittlerweile mit härtesten Sanktionen rechnen müssen. Besonders bedenklich war in diesem Zusammenhang der verfássungswidrige Einsatz der Bundeswehr in Heiligendamm, zeigte er doch die ideologische, institutionelle und ein satzpraktische Zusammenführung äuBerer und innerer Sicherheitspolitik in der "freien Wildbahn".

In Italien schickt die neue Berlusconi-Regierung Militärstreifen - und nicht nur wie bisher Carabinieri - zu sozialen Brennpunkten und lässt ihre Abschiebelager neuerdings vom Militär bewachen. In Griechenland, Italien und in Frankreich wurden in den vergangenen beiden Jahren erstmals Menschen, die an Ausschreitungen während Demos beteiligt gewesen sein sollen, in den Zusammenhang mit Terrorismus gestellt und $\mathrm{zu}$ hohen Haftstrafen verurteilt Und in den USA identifizierte der von der US-Regierung in Auftrag gegebene RAND-Bericht aus dem Jahr 2005 "drei innere terroristische Bedrohungen in den Vereinigten Staaten: AnarchistInnen, rechtsgerichtete ExtremistInnen und UmweltschutzaktivistInnen".2 Damit steht demnächst möglicherweise die Ausdeh-

2 Gene Ray: AktivistInnen im Visier. Der „Krieg gegen den Terror" und seine zusätzlichen Agenden, http:/transform.eipcp.net/ correspondence $/ 1202292557 /$ ?lid $=1204555355$ nung des "Feindstrafrechts", das für ausländische Gefangene in Guantánamo und anderen geheimen Gefängnissen bereits Praxis ist, auf AktivistInnen mit US-amerikanischer Staatsbürgerschaft ins Haus.

Die Logik des Feindstrafrechts ist auch schon in der bundesdeutschen Rechtsausübungspraxis eingeführt: eben im Begriff des "Gefährders". Seit 2004 wird diese Konstrulktion bei der Arbeitsgemeinschaft der Leiter der Landeskriminalämter und des Bundeskriminalamts verwendet. Die Katalogdelikte bestimmten diese $\mathrm{Be}$ hördenvertreter per Verweis auf den Paragraphen 100a der StPO, der eigentlich die Katalogstraftaten für die Zulässigkeit von Telekommunikationsübcrwachung listet. Paragraph $100 \mathrm{a}$ alleine verweist auf mindestens 39 Deliktgruppen aus 11 verschiedenen Einzelgesetzen. Die genauen Zahlen sind kaum exalkt feststellbar, da Mehrfachquerverweise innerhalb der Deliktgruppen auf weitere Gesetze verweisen, und der Katalog sich dadurch nicht nur beinahe unabsehbar ausdifferenziert, sondern auch weiter verbreitert. Mit dem unbestimmten Quasi-Rechtsbegriff des Gefährders ist die Quasi-Rechtsgrundlage dafür in der Welt, dass die Staatsicherheitsbehörden nahezu jeden und jede als Gefährder behandeln dürfen (und nicht nur können, sondern geradezu müssen).

Wieweit das auf Carl Schmitt zurückgehende Freund-Feind Denken bereits Eingang in die aktuelle Staatsrechtslehre gefunden hat, macht $\mathrm{Da}$ vid Salomon in seinem Artikel über das Werk des Kölner Staatsrechtsprofessors Otto Depenheuer deutlich. Mit seinem katholischen NeoKonservativismus ist Depenheuer der rechtsphilosophische Stichwortgeber maßgeblicher Sicherheits- und Innenpolitiker, wie aus Äußerungen etwa des Bundesinnenministers Wolfgang Schäuble deutlich hervorgeht. Dieser fordert die Bereitschaft der Zivilbevölkerung, sich als „Bürgeropfer $^{\text {" }}$ (Depenheuer) in einem von Terroristen gekaperten Flugzeug abschießen zu lassen. Norbert Geis (CSU) wünscht sich in einem Deutschlandfunk-Interview vom 9.7. 2008 die Einfuhrung von Telekommunikationsverboten und Sicherheitsverwahrung (also: politischer Lagerhaft) für Gefährder: „Aber ich glaube, dass dann, wenn der Gefährder wirklich erkannt ist, ich halte es für unverantwortlich, diesen Gefährder weiter unter uns ganz zwanglos leben zu lassen. (...) Er muss also in Sicherungsverwahrung gebracht werden. (...) In diesem Fall ohne Prozess. Das geht schwer runter, das sage ich Ihnen. (...) Aber es geht ja uns darum, diese freiheitliche Grundordnung zu schützen. Und deswegen wehren wir uns gegen die Gefährder. Und wir können nicht warten, bis die Gefährder zuschlagen." In den extra-territorialen Gefangenenlagern der USA ist dieser bürgerrechtliche Albtraum längst Programm. So äuBerte der Pentagon Sprecher Geoff Morrell kürzlich, die USA würden einige Guantánamo-Gefangene selbst dann nicht freilassen, wenn diese von einem Gericht freigesprochen wer- den: Sie stellten „eine Gefahr für die Welt dar" (Basler Zeitung, 7.8.2008). Der CSU-Politiker Geis will die Diskussion freilich noch weiter treiben: "Ich fordere eine Diskussion darüber, einmal, wie wir umgehen mit der gezielten Tötung eines potenziellen Aggressors und wie wir das gesetzlich, grundgesetzlich absichern können." Rechtsphilosophisch ist das durchaus konsistent: Auch eine solche „Kill-Fahndung" läge innerhalb der Logik, Legalität und Legitimität des neo-konservativen Feind- und Gefährder-Rechts.

Auch wenn sich eine qualitativ neue Politik mit der Inneren (Un-)Sicherheit noch nicht eindeutig diagnostizieren lässt, so gibt es doch zahlreiche Hinweise darauf, dass sie zumindest auf der politischen Agenda steht. In Deutschland wird die Reform des Versammlungsgesetzes auf Länderebene zum nächsten Prüfstein dieser Entwicklung. So stellt der bayerische Vorschlag das Grundrecht auf Versammlungsfreiheit vollends unter hoheitlichen Vorbehalt und kommt damit seiner Abschaffung gleich. Die faktische Abschaffung des Grundrechts auf Asyl wiederum jährt sich 2008 bereits zum 15. (!) Mal. Der Abbau von Schutzrechten sowie die Ausweitung staatlicher Zugriffskompetenzen werden vehement vorangetrieben. Möglicherweise wird es zum zweifelhaften historischen Verdienst der rechten Demokraten zählen, das Grundgesetz qualitativ geändert zu haben - damit lägen sie freilich „nur ${ }^{66}$ im internationalen Trend und agierten in der Logik neoliberaler Trans- 
formation. Eine grundlegend andere Sicherheitspolitik ist dagegen allein im Zuge einer grundlegend anderen Wirtschafts-, Außen- und Sozialpolitik denkbar. Um der Entwicklung eine andere Richtung zu geben, reicht freilich nicht ein dem Grundgesetz verpflichtetes Gericht. Dazu braucht es vielmehr starke linke soziale Bewegungen auf allen Politikfeldern, die sich nicht nur national zusammensetzen, sondern global für soziale Rechte und Menschenrechte kämpfen und mit ihrer "Kritik im Handgemenge“ (so David Salomon mit Marx) den staatlich-autoritären Tendenzen immer wieder in den Arm fallen.

$$
* * *
$$

Außerhalb des Schwerpunkts setzt Jürgen Link mit seinem Artikel zu den Stärken und Schwächen von Foucaults Marx-Kritik die in unserem letzten Heft (PROKLA 151: Gesellschaftstheorie nach Marx und Foucault, Juni 2008) begonnene Debatte fort. Der zweite Beitrag außerhalb des Schwerpunkts stammt von dem chinesischen Historiker und Philosophen Wang Hui. Inzwischen sind auch in China Ansätze einer eigenständigen linken Debatte, jenseits des parteioffiziellen Marxismus entstanden, Wang Hui ist einer der Protagonisten dieser Debatte. Sein Blick auf die Entwicklung Chinas nach der Kulturrevolution und dabei zu Tage tretende Ähnlichkeiten mit dem politischen System der westlichen Länder ist gleichermaßen ungewohnt wie aufschlussreich.

Stephan Lessenich geht in seinem Einspruch auf die reflexhaften Reaktionen ein, die der im Frühsommer veröffentlichte 3. Armutsbericht der Bundesregierung auf konservativer Seite ausgelöst hat. Slave Cubcla setzt sich in seinem Beitrag kritisch mit PROKLA 150, Umkämpfte Arbeit (März 2008) auseinander, wobei es ihm weniger um Defizite in einzelnen Artikeln, als vielmehr um die gesellschaftlichen Hintergründe der Ausblendung bestimmter Fragestellungen.

$$
* * *
$$

An der Konzeption und Gestaltung des Schwerpunkts dieser Ausgabe wirkte Markus Euskirchen als Gastredakteur mit. Für sein Engagement, seine Ideen und seine Anregungen möchten wir uns ganz herzlich bei ihm bedanken.

\section{Andreas Fisahn}

\section{Repressive Toleranz und der "Pluralismus" der Oligarchien}

Der „nationale Sicherheitsrat“ lässt sich gleichsam als Allegorie für die neuere Diskussion um Sicherheitsgesetze, Überwachung und die Einschränkung grundrechtlicher Freiheiten verstehen. Die Diskussion begann unter neuem Vorzeichen mit dem 11. September 2001, nahm an Fahrt aber erst auf im Sommer 2007 nach dem massiven Auftreten der Sicherheitsbehörden und Apparate in Heiligendamm. Der Sicherheitsrat steht für unterschiedliche Elemente der Sicherheitspolitik. Es lässt sich konstatieren eine informationelle Aufrüstung des Staates, die sich die neueren technischen Möglichkeiten der Überwachung und Generierung von Informationen zu Nutze macht. Er steht für tatsächliche und geplante Verschiebungen und Veränderungen der Kompetenzen von Polizei, Geheimdiensten und Bundeswehr. Er steht damit für eine Bündelung staatlicher Macht, deren rechtliche Begrenzung auf Schwierigkeiten stößt, d.h. für einen strategischen Umgang der Sicherheitsbehörden mit den rechtlichen Schranken, wie sie nicht zuletzt in der Folter-Diskussion sich äußert. Er steht für die Wiederkehr eines Feinddenkens, das in ein Feindrecht gegenüber anderen, Fremden, gegenüber der Bedrohung eines "Außen" münden soll. Diese verschiedenen Elemente einer - nicht im Ganzen neuen - „Sicherheitspolitik" sollen hier nicht ausführlich nachgezeichnet werden. Vielmehr wird der Versuch unternommen, diese Elemente oder Tendenzen mit ihren Gegentendenzen zu erfassen, um mögliche Entwicklungen und Gefahren in den Blick zu bekommen. Dazu sollen (1) grundlegende Spannungslinien der Gesellschaft skizziert werden, welche (2) den theoretischen Einordnungen von Veränderungen des Rechtsstaates und der Demokratie zugrunde lagen. Im Anschluss wird (3) die Entwicklung einer kontinuierlichen informationellen Aufrüstung nachgezeichnet und ihre ambivalente Bedeutung diskutiert. Sie wird unterschieden von (4) einer Politik repressiver Unterdrückung sozialer Proteste und oppositioneller Strömungen; auch in diesem Feld muss zwischen einer integrierten Mehrheitsgesellschaft und ausgegrenzten sowie abgehängten Teilen unterschieden werden. Schließlich wird (5) eine Strategie der Oligarchisierung von Entscheidungsstrukturen als Antwort auf die soziale Desintegration in ihren unterschiedlichen Dimensionen eher angedeutet als umfassend erörtert. 